Pacific Journal of Mathematics

CLASSES OF DEFINITE GROUP MATRICES 


\title{
CLASSES OF DEFINITE GROUP MATRICES
}

\author{
R. C. ThOMPSON
}

Two positive definite symmetric $n \times n$ matrices $A, B$ with integer elements and determinant one are said to be congruent if there exists an integral $C$ such that $B=C A C^{T}\left(C^{T}\right.$ is the transpose of $C$ ). This is an equivalence relation. The number of equivalence classes, $C$-classes, is finite and is known for all $n \leqq 16$. Let $G$ be a finite group of order $n$ and let $Y, Z$ be two positive definite symmetric group matrices for $G$ with integral elements and determinant one. If an integral group matrix $X$ for $G$ exists such that $Z=X Y X^{T^{\prime}}$ then $Z, Y$ are said to be $G$-congruent. $G$ congruence is an equivalence relation. In this paper the interlinking of the $G$-classes with the $C$-classes is determined for all groups of order $n \leqq 13$. The principal result is that the $G$-class number is two for certain groups of orders eight or twelve and is one for all other groups of order $n \leqq 13$.

Let $G$ be a finite group with elements $g_{1}, g_{2}, \cdots, g_{n}$. Let $x_{1}, x_{2}, \cdots, x_{n}$ be variables and let $X$ be an $n \times n$ matrix whose $(i, j)$ element is $x_{k}$ where $k$ is determined by $g_{k}=g_{i} g_{j}^{-1}$. We say $X$ is a group matrix for $G$. In this paper we study group matrices which have rational integers as elements. We call a matrix $M$ integral if its elements are rational integers, unimodular if the determinant of $M=\operatorname{det} M= \pm 1$, symmetric if $M=M^{T}$ where $M^{T}$ is the transpose of $M$. We let $M^{*}$ denote the complex conjugate of $M^{T}$. The words positive, definite, symmetric, integral, unimodular are abbreviated as $p, d, s, i, u$, respectively. We say pdsiu matrices $M$ and $M_{1}$ are congruent if $M_{1}=$ $U M U^{T}$ for some $i u U$. Congruence is an equivalence relation on the set of $n \times n$ pdsiu matrices. The number of equivalence classes (briefly: $C$-classes) is finite and in fact [2] is one for $1 \leqq n \leqq 7$, two for $8 \leqq n \leqq 11$, and three for $n=12,13$. If $G$ is a finite group we say pdsiu group matrices $M$ and $M_{1}$ are $G$-congruent if $M_{1}=U M U^{T}$ for some iu group matrix $U$ for $G$. Since sums, products, inverses, and transposes of group matrices for $G$ are still group matrices for $G, G$ congruence is an equivalence relation on the set of pdsiu group matrices for $G$. Not much is known about the equivalence classes (briefly: G-classes). In this paper we find all $G$-classes and determine their relationship with the $C$-classes for all groups of order $n \leqq 13$; we also get a little information for $n>13$. Our interest in this problem stems from the following Theorem 1, proved in [8].

Received November 9, 1964. 
THEOREM 1. If a pdsiu group matrix $M$ for $G$ is in the principal $C$-class then $M$ is in the principal G-class, when $G$ is solvable.

The principal class is, of course, the class containing $I_{n}$, the $n \times n$ identity matrix.

One may ask: are there any pdsiu group matrices for $G$, other than the identity?

THEOREM 2. There exist pdsiu group matrices for $G$ in addition to the identity precisely when $G$ is not any of the following types of groups:

(i ) the direct product of cyclic groups of orders two and/or four;

(ii) the direct product of cyclic groups of orders two and/or three;

(iii) the quaternion group or the direct product of the quaternion group with cyclic groups of order two.

Proof. Combining the discussion on p. 340 of [6] with Theorem 11 of [1] shows that an iu group matrix for $G$ exists which is not a permutation matrix or the negative of a permutation matrix precisely when $G$ is not any of the groups (i), (ii), (iii). If $M$ is an iu group matrix for $G$, not a permutation matrix or the negative of a permutation matrix, then $M M^{T}$ is a pdsiu group matrix for $G$ and not the identity since the $(i, i)$ element of $M M^{T}$ is the sum of squares of the integers in row $i$ of $M$.

Concerning the finiteness of the $G$-class number, only the following fact is known.

THEOREM 3. The $G$ class number is finite if $G$ is abelian.

Proof. This follows from the argument of [3], making use of Lemma 2 of [7].

2. Two lemmas. Let $P=P_{n}$ be the $n \times n$ companion matrix of the polynomial $\lambda^{n}-1$. Let $v=v_{n}=(1,1, \cdots, 1)$ be the row $n$-tuple in which each entry is one.

LEMMA 1. Let $p$ be an odd prime and let $t$ be an integer prime to $p$. Then $\lambda=1$ is a simple eigenvalue of $P_{p}^{t}, \lambda=-1$ is not an eigenvalue, and $v_{p}$ spans the eigenspace of $P_{p}^{t}$ belonging to $\lambda=1$.

Proof. The eigenvalues of $P_{p}$ are 1 and the $p-1$ primitive $p$ th roots of unity. Hence this is also true of $P_{p}^{t}$ since $\omega^{t}$ is a primitive $p$ th root of unity if $\omega$ is and $(t, p)=1$. Thus 1 is a simple eigenvalue of $P_{p}^{t}$ and -1 is not an eigenvalue. Since $v_{p} P_{p}=v_{p}$, the last assertion is immediate. 
Let $\bar{\alpha}$ denote the complex conjugate of $\alpha$.

LeMMA 2. Let

$$
\left(\begin{array}{ll}
\alpha & \bar{\beta} \\
\beta & \bar{\alpha}
\end{array}\right)\left(\begin{array}{ll}
x & \bar{y} \\
y & x
\end{array}\right)\left(\begin{array}{ll}
\bar{\alpha} & \bar{\beta} \\
\beta & \alpha
\end{array}\right)=\left(\begin{array}{ll}
x_{1} & \bar{y}_{1} \\
y_{1} & x_{1}
\end{array}\right)
$$

where $\alpha, \beta, y$ are complex numbers and $x$ is a positive real number. Let $x^{2}-|y|^{2}=1$. If $|\alpha|^{2}-|\beta|^{2}=1$ then $x_{1}<x$ implies $|\beta|<|y|$ and $x_{1} \leqq x$ implies $|\beta| \leqq|y|$. If $|\alpha|^{2}-|\beta|^{2}=-1$ then $x_{1}<x$ implies $|\alpha|<|y|$ and $x_{1} \leqq x$ implies $|\alpha| \leqq|y|$.

Proof. The cases $\alpha=0$ or $\beta=0$ are easy. Let $\alpha \neq 0, \beta \neq 0$, $|\alpha|^{2}-|\beta|^{2}=1$. Now $|\alpha|^{2}+|\beta|^{2}=1+2|\beta|^{2}$, hence $x_{1}-x=2 x|\beta|^{2}+$ $y \bar{\alpha} \bar{\beta}+\bar{y} \alpha \beta<0$ if $x_{1}<x$. Hence $0<2 x|\beta|^{2}<-y \bar{\alpha} \bar{\beta}-\bar{y} \alpha \beta$. By the triangle inequality we get $2 x|\beta|^{2}<2|y||\alpha||\beta|$, hence $x^{2}|\beta|^{2}<|y|^{2}|\alpha|^{2}=$ $|y|^{2}\left(1+|\beta|^{2}\right)$, therefore $\left(x^{2}-|y|^{2}\right)|\beta|^{2}<|y|^{2}$, or $|\beta|<|y|$ as required. A similar computation holds when $x_{1} \leqq x$ or when $|\alpha|^{2}-|\beta|^{2}=-1$.

An $n \times n$ circulant is, by definition, a polynomial in $P_{n}$. It is also a group matrix for the cyclic group of order $n$. Since $P_{n}$ is unitarily diagonable, given a circulant

$$
X=\sum_{i=0}^{n-1} x_{i} P_{n}^{i},
$$

there exists a unitary $V$, independent of $X$, such that $V X V^{*}=$ $\operatorname{diag}\left(\xi_{0}, \xi_{1}, \cdots, \xi_{n-1}\right)$ where

$$
\xi_{i}=\sum_{j=0}^{n-1} x_{j} \omega^{i j},
$$$$
0 \leqq j \leqq n-1
$$

Here $\omega$ is a primitive $n$th root of unity. We make frequent use of this fact. If $Y=\left(Y_{i j}\right)$ is partitioned into blocks $Y_{i j}$ each of which is a circulant and if $W=V+V+\cdots+V$ ( + denotes direct sum) then each of the blocks in $W Y W^{*}$ is diagonalized. One may find a permutation matrix $Q$ for which $Q W Y W^{*} Q^{*}$ splits into a direct sum. In the computations of $\S \S 4-9$ some of the direct summands will again be circulants and so may themselves be unitarily diagonalized. In this manner we obtain the unitary $U$ and the irreducible constituents of the group matrices of $\S \S 4-9$. We also use the fact that a circulant equation like $Z=X Y$ holds if and only if $\xi_{i}(Z)=\xi_{i}(X) \xi_{i}(Y)$ for all $i$.

3. The $C$-classes $\Phi_{r}+I_{j}$, where $\Phi_{r}$ does not represent one. Let $\Phi_{r}$ be an $r \times r$ pdsiu matrix (not necessarily a group matrix) such that $x \Phi_{r} x^{T} \neq 1$ for any integral vector $x$. 
THEOREM 4. The $C$-class of $\Phi_{r}+I_{j}$ does not contain any group matrix if there exists an odd prime divisor $p$ of $r+j$ which does not divide $r$.

Proof. Let $n=r+j$. Since $\Phi_{r}$ does not represent one, it is easy to find all integral $n$-tuples $x$ for which $x\left(\Phi_{r}+I_{j}\right) x^{T}=1$. The number of such $x$ is exactly $2 j$. Suppose $X$ is a group matrix for some group $G$, with $X$ in the $C$-class of $\Phi_{r}+I_{j}$. Then $G$ contains an element $a$ of order $p$. Let $H$ be the cyclic subgroup of $G$ generated by $a$ and let $g_{1} H, g_{2} H, \cdots, g_{k} H,(k=n / p)$, be the cosets of $H$ in $G$. If we take the elements of $G$ in the order $g_{1}, g_{1} \alpha, g_{1} a^{2}, \cdots, g_{1} a^{p-1}, g_{2}, g_{2} \alpha, g_{2} a^{2}, \cdots$, $g_{2} a^{p-1}, \cdots, g_{k}, g_{k} a, g_{k} a^{2}, \cdots, g_{k} a^{p-1}$, then the group matrix $X$ partitions as $X=\left(X_{i j}\right)_{1 \leqq i, j \leq k}$, where each $X_{i j}$ is a $p \times p$ circulant. If $Q=P_{p} \dot{+}$ $P_{p}+\cdots+P_{p}$ then $Q X Q^{T}=X$. Let $x=\left(x_{1}, x_{2}, \cdots, x_{k}\right)$ be a row $n$-tuple, where each $x_{i}$ is a row $p$-tuple. If $x$ is integral and $x X x^{T}=1$ then $\left(x Q^{\alpha}\right) X\left(x Q^{\alpha}\right)^{T}=1$ for $\alpha=0,1,2, \cdots, p-1$. If $x Q^{\alpha}=x Q^{\beta}$ for a pair $\alpha$, $\beta$ with $0 \leqq \beta<\alpha<p$ then $x Q^{\alpha-\beta}=x$. This implies $x_{i} P_{p}^{\alpha-\beta}=x_{i}$ for $1 \leqq i \leqq k$, and by Lemma $1, x_{i}=\lambda_{i} v_{p}, 1 \leqq i \leqq k$. Since $x_{i}$ is integral, $\lambda_{i}$ is an integer. Moreover, $v_{p}$ is an eigenvector of $P_{p}$, hence of any $p \times p$ circulant, hence $v_{p} X_{i j}=r_{i j} v_{p}$. Here $r_{i j}$ is an integer (in fact the sum down any column of $X_{i j}$ ). Now

$$
\begin{aligned}
x X x^{T} & =\sum_{i, j=1}^{l} x_{i} X_{i j} x_{j}^{T^{\prime}} \\
& =\sum_{i, j=1}^{l} \lambda_{i} \lambda_{j} r_{i j} p \\
& \equiv 0(\bmod p)
\end{aligned}
$$

because $v_{p} v_{p}^{T}=p$. This contradicts $x X x^{T}=1$, hence $x Q^{\alpha}=x Q^{\beta}$ is impossible. If $x Q^{\alpha}=-x Q^{\beta}$ then $x Q^{\alpha-\beta}=-x$, so $x_{i} P_{p}^{\alpha-\beta}=-x_{i}, 1 \leqq$ $i \leqq k$. By Lemma 1 this implies $x_{i}=0$. Hence $x=0$, a clear falsehood. Thus $\pm x Q^{\alpha}$ for $0 \leqq \alpha<p$ are $2 p$ distinct integral solutions of $y X y^{T}=1$. If $y$ is further solution then $\pm y Q^{\alpha}, 0 \leqq \alpha<p$ are also all different. If $\pm y Q^{\alpha}= \pm x Q^{\beta}$ then $y= \pm x Q^{\gamma}$, for some $\gamma, 0 \leqq \gamma<p$, and this contradicts the choice of $y$. Thus the integral vectors representing one come in nonoverlapping sets of $2 p$. We thus have $j \equiv 0(\bmod p)$. Since $r+j \equiv 0(\bmod p)$, we get $r \equiv 0(\bmod p)$, a contradiction.

Now let $\Phi_{n}($ for $n \equiv 0(\bmod 4), n>4)$ be the matrix on p. 331 of [5]. Then it is known that $\Phi_{n}$ is pdsiu and $\Phi_{n}$ does not represent one. Representatives of the nonprincipal $C$-classes up to $n=13$ are $\Phi_{8}, \Phi_{8}+I_{j}$ for $1 \leqq j \leqq 5, \Phi_{12}, \Phi_{12}+I_{1}$. 
CoRollary. The only non principal $n \times n$ C-classes for $n \leqq 13$ that can contain a group matrix are the C-classes of $\Phi_{8}$ and $\Phi_{12}$.

4. The dihedral group of order eight. The dihedral group of order $2 n$ is generated by two elements $a, b$ with $a^{n}=b^{2}=1, b^{-1} a b=a^{-1}$. If we take the elements in the order $1, a, a^{2}, \cdots, a^{n-1}, b, b a, b a^{2}, \cdots, b a^{n-1}$, then the group matrix $X$ has the form

$$
X=\left(\begin{array}{ll}
A & C \\
B & D
\end{array}\right)
$$

where $A, B, C, D$ are $n \times n$ circulants and $C=B^{T}, D=A^{T}$. If $n=4$ and $A=x_{0} I+x_{1} P+x_{2} P^{2}+x_{3} P^{3}, B=x_{4} I+x_{5} P+x_{6} P^{2}+x_{7} P^{3}$, then there exists a unitary $U$ such that $U X U^{*}=\left(\varepsilon_{1}\right)+\left(\varepsilon_{2}\right)+\left(\varepsilon_{3}\right)+\left(\varepsilon_{4}\right)+$ $X_{1}+X_{1}$ where:

$$
\begin{gathered}
\frac{1}{2}\left[\begin{array}{c}
\varepsilon_{1} \\
\varepsilon_{2} \\
\varepsilon_{3} \\
\varepsilon_{4}
\end{array}\right]=\left[\begin{array}{rrrr}
\frac{1}{2} & \frac{1}{2} & \frac{1}{2} & \frac{1}{2} \\
\frac{1}{2} & \frac{1}{2} & -\frac{1}{2} & -\frac{1}{2} \\
\frac{1}{2} & -\frac{1}{2} & \frac{1}{2} & -\frac{1}{2} \\
\frac{1}{2} & -\frac{1}{2} & -\frac{1}{2} & \frac{1}{2}
\end{array}\right]\left[\begin{array}{l}
\eta_{1} \\
\eta_{2} \\
\eta_{3} \\
\eta_{4}
\end{array}\right] \\
\eta_{1}=x_{0}+x_{2}, \eta_{2}=x_{1}+x_{3}, \eta_{3}=x_{4}+x_{6}, \eta_{4}=x_{5}+x_{7}, \\
X_{1}=\left[\begin{array}{ll}
A_{X}+i B_{X} & C_{X}-i D_{X} \\
C_{X}+i D_{X} & A_{X}-i B_{X}
\end{array}\right], \\
A_{X}=2 x_{0}-\eta_{1}, B_{X}=2 x_{1}-\eta_{2}, C_{X}=2 x_{4}-\eta_{3}, D_{X}=2 x_{5}-\eta_{4} .
\end{gathered}
$$

For $X$ to be $i u$ each of $\varepsilon_{1}, \varepsilon_{2}, \varepsilon_{3}, \varepsilon_{4}$, det $X_{1}$ must be \pm 1 since each of these is a rational integer. Since the matrix in (3) is unitary,

$$
\eta_{1}^{2}+\eta_{2}^{2}+\eta_{3}^{2}+\eta_{4}^{2}=\left(\left|\varepsilon_{1}\right|^{2}+\left|\varepsilon_{2}\right|^{2}+\left|\varepsilon_{3}\right|^{2}+\left|\varepsilon_{4}\right|^{2}\right) / 4=1
$$

Consequently as $\eta_{1}, \eta_{2}, \eta_{3}, \eta_{4}$ are rational integers, exactly one of $\eta_{1} \eta_{2}, \eta_{3}, \eta_{4}$ is \pm 1 , and the other three are zero. Thus exactly one of $A_{X}, B_{X}, C_{X}, D_{X}$ is odd, the other three are even. From $\operatorname{det} X_{1}= \pm 1$ we get $\operatorname{det} X_{1}=1$ if $A_{X}$ or $B_{X}$ is even, $\operatorname{det} X_{1}=-1$ if $C_{X}$ or $D_{X}$ is even. (Consider $A_{X}^{2}+B_{X}^{2}-C_{X}^{2}-D_{X}^{3}= \pm 1$ modulo 4.) Conversely if $A_{X}, B_{X}, C_{X}, D_{X}$ are integers, one even, three odd, with $A_{X}^{2}+B_{X}^{2}-$ $C_{X}^{2}-D_{X}^{2}= \pm 1$ we can use (3), (4), (5), (6) to construct an iu group matrix $X$. The pdsiu group matrices arise when $\varepsilon_{1}=\varepsilon_{2}=\varepsilon_{3}=\varepsilon_{4}=$ $\eta_{1}=1, A_{X}>0$. 
Now let $Y, Z$ be pdsiu group matrices. Then $Z=X Y X^{T}$ holds if and only if $U Z U^{*}=\left(U X U^{*}\right)\left(U Y U^{*}\right)\left(U X U^{*}\right)^{*}$; and this holds if and only if $Z_{1}=X_{1} Y_{1} X_{1}^{*}$, and $\varepsilon_{\imath}(Z)=\varepsilon_{i}(X) \varepsilon_{i}(Y) \overline{\varepsilon_{i}(X)}$, for $i=1,2,3,4$. This last condition is satisfied since the $\varepsilon_{i}(X)$ are \pm 1 . Here, and henceforth, let $\rho_{1}, \rho_{2}, \rho_{3}, \rho_{4}$ stand for integers which may independently be \pm 1 . We now use a descent argument. We attempt to choose $A_{X}, B_{X}, C_{X}, D_{X}$ so that $A_{Z}<A_{Y}$. As in the proof of Lemma 2, we have

$$
\begin{aligned}
\left(A_{Z}-A_{Y}\right) / 2= & A_{Y}\left(C_{X}^{2}+D_{X}^{2}\right) \\
& +C_{Y}\left(A_{X} C_{X}-B_{X} D_{X}\right)+D_{Y}\left(A_{X} D_{X}+B_{X} C_{X}\right) .
\end{aligned}
$$

Put $A_{X}=\rho_{1}, B_{X}=2 \rho_{2}, C_{X}=2 \rho_{3}, D_{X}=0$. Then $X$ is $i u$ and by (8) we can choose the signs $\rho_{1}, \rho_{2}, \rho_{3}$ so that $A_{Z}<A_{Y}$ if

$$
2 A_{Y}-\left|C_{Y}\right|-2\left|D_{Y}\right|<0 \text {. }
$$

Next take $A_{X}=\rho_{1}, B_{X}=2 \rho_{2}, C_{X}=0, D_{X}=2 \rho_{4}$. Then $X$ is $i u$ and by (8) we may choose the signs $\rho_{1}, \rho_{2}, \rho_{4}$ so that $A_{Z}<A_{Y}$ if

$$
2 A_{Y}-2\left|C_{Y}\right|-\left|D_{Y}\right|<0 \text {. }
$$

Since $A_{Y}^{2}=1+C_{Y}^{2}+D_{Y}^{2}, A_{Y}>0$, (9) holds

$$
\begin{array}{ll}
\Leftrightarrow & 2 A_{Y}<\left|C_{Y}\right|+2\left|D_{Y}\right|, \\
\Leftrightarrow & 4 A_{Y}^{2}<C_{Y}^{2}+4\left|C_{Y} D_{Y}\right|+4 D_{Y}^{2}, \\
\Leftrightarrow & 4\left(1+C_{Y}^{2}+D_{Y}^{2}\right)<C_{Y}^{2}+4\left|C_{Y} D_{Y}\right|+4 D_{Y}^{2}, \\
\Leftrightarrow & 4+3 C_{Y}^{2}-4\left|C_{Y}\right|\left|D_{Y}\right|<0 .
\end{array}
$$

Similarly (10) holds if and only if

$$
4+3 D_{Y}^{2}-4\left|C_{Y}\right|\left|D_{Y}\right|<0 .
$$

Now the region in the positive quadrant of the $C_{Y}, D_{Y}$ plane not satisfying either (11) or (12) is a region of infinite extent with a portion of two hyperbolas as part of the boundary. The only points in this region with even integral coordinates have either $C_{Y}=0$ or $D_{Y}=0$, or else $\left|C_{Y}\right|=\left|D_{Y}\right|=2$. Now if $C_{Y}=0$ we get from $A_{Y}^{2}=$ $1+C_{Y}^{2}+D_{Y}^{2}$ that $\left(A_{Y}-D_{Y}\right)\left(A_{Y}+D_{Y}\right)=1$, so $A_{Y}+C_{Y}=A_{Y}-C_{Y}=$ \pm 1 , hence $A_{Y}=1, D_{Y}=0$. Now $A_{Y}=1, C_{Y}=D_{Y}=0$ gives $Y=I_{8}$. Thus any pdsiu group matrix $Y$ is in the same $G$-class as $I_{8}$ or clse in the $G$-class of a $Y$ for which $C_{Y}= \pm 2, D_{Y^{\prime}}= \pm 2, A_{Y}=3$. That these last four possible $Y$ are in the same $G$-class is seen as follows. Let $T$ denote the pdsiu group matrix with $A_{T}=3, C_{T}=2, D_{T}=2$. If $A_{X}=3, B_{X}=0, C_{X}=-2, D_{X}=-2$ then $Z=X T X^{T}$ has $A_{Z}=3$, $B_{z}=0, C_{z}=-2, D_{z}=-2$. If $A_{X}=-2, B_{X}=-2, C_{X}=3, D_{X}=0$ then $Z=X T X^{t}$ has $A_{Z}=3, B_{z}=0, C_{Z}=-2, D_{z}=2$. If $A_{X}=2$, 
$B_{X}=-2, C_{X}=0, D_{X}=-3$ then $Z=X T X^{T}$ has $A_{Z}=3, B_{Z}=0, C_{Z}=2$, $D_{Z}=-2$. Thus the $G$-class number is $\leqq 2$. If it were one there would be an $X$ such that $X_{1} T_{1} X_{1}^{*}=I_{2}$. Lemma 2 then shows that if $\operatorname{det} X_{1}=1$ we have $C_{X}^{2}+D_{X}^{2}<C_{T^{\prime}}^{2}+D_{T}^{2}=8$ and if $\operatorname{det} X_{1}=-1$ then $A_{X}^{2}+B_{X}^{2}<8$. All possible $A_{X}, B_{X}, C_{X}, D_{X}$ are easily found and none work.

5. The other groups of order eight. The cyclic group of order eight is completely worked out in [4]. The $G$ class number is two. The only pdsiu group matrix belonging to any of the remaining groups of order eight is $I_{8}$.

6. The cyclic group of order twelve. Let $X=x_{0} I_{12}+x_{12} P_{12}+$ $\cdots+x_{11} P_{12}$. Take $\omega=\left(3^{1 / 2}+i\right) / 2$ for the primitive root of unity of order twelve. Then for a unitary $U, U X U^{*}=\operatorname{diag}\left(\xi_{0}, \xi_{1}, \cdots, \xi_{11}\right)$ where (see (1)):

$$
\begin{aligned}
& {\left[\begin{array}{rrrr}
1 / 2 & 1 / 2 & 1 / 2 & 1 / 2 \\
1 / 2 & i / 2 & -1 / 2 & -i / 2 \\
1 / 2 & -1 / 2 & 1 / 2 & -1 / 2 \\
1 / 2 & -i / 2 & -1 / 2 & i / 2
\end{array}\right]\left[\begin{array}{l}
\eta_{0} \\
\eta_{3} \\
\eta_{6} \\
\eta_{9}
\end{array}\right]=\frac{1}{2}\left[\begin{array}{l}
\xi_{0} \\
\xi_{3} \\
\xi_{6} \\
\xi_{9}
\end{array}\right]} \\
& \eta_{0}=x_{0}+x_{4}+x_{8}, \eta_{3}=x_{1}+x_{5}+x_{9}, \eta_{6}=x_{2}+x_{6}+x_{10}, \\
& \eta_{9}=x_{3}+x_{7}+x_{11} \\
& \xi_{1}=\left[2 x_{0}+x_{2}-x_{4}-2 x_{6}-x_{8}+x_{10}\right. \\
& +i\left(x_{1}+2 x_{3}+x_{5}-x_{7}-2 x_{9}-x_{11}\right) \\
& \left.+3^{1 / 2}\left(x_{1}-x_{5}-x_{7}+x_{11}\right)+(-3)^{1 / 2}\left(x_{2}+x_{4}-x_{8}-x_{10}\right)\right] / 2, \\
& \xi_{2}=\left[2 x_{0}+x_{1}-x_{2}-2 x_{3}-x_{4}+x_{5}+2 x_{6}+x_{7}-x_{8}-2 x_{9}\right. \\
& \left.-x_{10}+x_{11}+(-3)^{1 / 2}\left(x_{1}+x_{2}-x_{4}-x_{5}+x_{7}+x_{8}-x_{10}-x_{11}\right)\right] / 2 \text {, } \\
& \xi_{4}=\left[2 x_{0}-x_{1}-x_{2}+2 x_{3}-x_{4}-x_{5}+2 x_{6}-x_{7}-x_{8}+2 x_{9}\right. \\
& \left.-x_{10}-x_{11}+(-3)^{1 / 2}\left(x_{1}-x_{2}+x_{4}-x_{5}+x_{7}-x_{8}+x_{10}-x_{11}\right)\right] / 2 \text {. }
\end{aligned}
$$

The remaining $\xi_{i}$ are conjugate to one of $\xi_{1}, \xi_{2}, \xi_{4}$ in the field $R(\omega)$ of the 12 th root of unity. As $\xi_{0}, \cdots, \xi_{11}$ are algebraic integers, $X$ is unimodular if and only if $\xi_{0}, \cdots, \xi_{11}$ are units. Since the matrix in (13) is unitary, $\eta_{0}^{2}+\eta_{3}^{2}+\eta_{6}^{3}+\eta_{9}^{2}=\left(\left|\xi_{0}\right|^{2}+\left|\xi_{3}\right|^{2}+\left|\xi_{6}\right|^{2}+\left|\xi_{9}\right|^{2}\right) / 4=1$ since $\xi_{0}, \xi_{3}, \xi_{6}, \xi_{9}$ are units in the Gaussian integers, hence roots of unity. As $\eta_{0}, \eta_{3}, \eta_{6}, \eta_{9}$ are rational integers, exactly one of $\eta_{0}, \eta_{3}, \eta_{6}, \eta_{9}$ is \pm 1 , the other three are zero. We now show that we can find a circulant $W$ of the form $\pm P_{12}^{\alpha}$ so that in $X W$ we have

$$
\eta_{0}=1=\xi_{0}=\xi_{3}=\xi_{6}=\xi_{9}
$$


and $\xi_{2}= \pm 1$. If, for $X, \eta_{0}= \pm 1$ then by (13), $\xi_{0}=\xi_{3}=\xi_{6}=\xi_{9}=\eta_{0}$ and for $X\left(\eta_{0} I_{12}\right),(18)$ is satisfied. If, for $X, \eta_{3}= \pm 1$, then by (13), $\xi_{0}=\eta_{3}, \xi_{3}=i \eta_{3}, \xi_{6}=-\eta_{3}, \xi_{9}=-i \eta_{3}$. Then, for $X\left(\eta_{3} P_{12}^{3}\right)$, (18) is satisfied. If, for $X, \eta_{6}= \pm 1$, then by (13), $\xi_{0}=\eta_{6}, \xi_{3}=-\eta_{6}, \xi_{6}=\eta_{6}, \xi_{9}=-\eta_{6}$. Then, for $X\left(\eta_{6} P_{12}^{2}\right)$, (18) is satisfied. If, for $X, \eta_{9}= \pm 1$, then by (13), $\xi_{0}=\eta_{9}, \xi_{3}=-i \eta_{9}, \xi_{6}=-\eta_{9}, \xi_{9}=i \eta_{9}$, and for $X\left(\eta_{9} P_{12}\right)$, (18) is satisfied. So now let $X$ satisfy (18). For $X, \xi_{2}$ is a unit in the field $R\left((-3)^{1 / 2}\right)$, hence $\xi_{2}$ is a power of $\omega^{2}=\left(1+(-3)^{1 / 2}\right) / 2$. We can choose $\lambda$ to be $-1,0$, or 1 , such that for $X P_{12}^{4 \lambda}$ we still have (18) and, moreover, $X P_{12}^{4 \lambda}$ has $\xi_{2}$ equal to $\omega^{0}$ or $\omega^{6}$; that is $\xi_{2}= \pm 1$. Thus we have achieved our claim. Note that $\xi_{4}$ is also a unit in $R\left((-3)^{1 / 2}\right)$ and that the rational part of the numerator of $\xi_{4}$ is congruent $(\bmod 2)$ to the rational part of the numerator of $\xi_{2}$. Since the only units in $R\left((-3)^{1 / 2}\right)$ are $\left( \pm 1 \pm(-3)^{1 / 2}\right) / 2$ or $\pm 2 / 2, \xi_{2}= \pm 1$ forces $\xi_{4}= \pm 1$.

We now construct the pdsiu circulants $X$. These have all $\xi_{i}$ real and positive, whence (18) holds. Symmetry implies $x_{11-j}=x_{1+j}$ for $0 \leqq j \leqq 4$. Then for the $\xi_{i}$ to be positive units we require $\xi_{0}=\xi_{2}=$ $\xi_{3}=\xi_{4}=\xi_{6}=1$, hence:

$$
\begin{aligned}
x_{0}+2 x_{1}+2 x_{2}+2 x_{3}+2 x_{4}+2 x_{5}+x_{6} & =1, \\
x_{0}+x_{1}-x_{2}-2 x_{3}-x_{4}+x_{5}+x_{6} & =1, \\
x_{0}-2 x_{2}+2 x_{4}-x_{6} & =1, \\
x_{0}-x_{1}-x_{2}+2 x_{3}-x_{4}-x_{5}+x_{6} & =1, \\
x_{0}-2 x_{1}+2 x_{2}-2 x_{3}+2 x_{4}-2 x_{5}+x_{6} & =1 .
\end{aligned}
$$

Solving these simultaneously we get $x_{0}=1-2 x_{4}, x_{5}=-x_{1}, x_{3}=0$, $x_{2}=-x_{4}, x_{6}=2 x_{4}$. Then $\xi_{1}=1-6 x_{4}+(3)^{1 / 2}\left(2 x_{1}\right)$, and $\xi_{1} \xi_{5}=\left(1-6 x_{4}\right)^{2}-$ $3\left(2 x_{1}\right)^{2}=1$ if $\xi_{1}, \xi_{5}$ are to be positive units. Hence $\xi_{1}$ satisfies a Pell's equation, the fundamental solution of which is $2-3^{1 / 2}$. Now by induction one easily checks that all odd powers of $2-3^{1 / 2}$ have even rational part and all even powers have rational part $\equiv 1(\bmod 6)$ and even irrational part. Consequently all pdsiu circulants are powers of the circulant $M$ for which $\eta_{0}=1=\xi_{0}=\xi_{3}=\xi_{6}=\xi_{9}=\xi_{2}=\xi_{4}, \xi_{1}=$ $\left(2-3^{1 / 2}\right)^{2}=7-4 \cdot 3^{1 / 2}$. Now $M^{2 \alpha}=M^{\alpha}\left(M^{\alpha}\right)^{T}$ is in the principal $G$-class and $M^{2 \alpha+1}=M^{\alpha} \cdot M \cdot\left(M^{\alpha}\right)^{T}$ is in the G-class of $M$. To show that the $G$-class number is two, we need only show that $M$ is not in the principal $G$-class. If $M=X X^{T}$ for $X$ an $i u$ circulant, then for any $W$ of the form $W= \pm P_{12}^{a}$ we have $M=(X W)(X W)^{T}$. Then by the remarks of the previous paragraph, we may, after changing $X W$ to $X$, assume that $M=X X^{r}$ where, for $X$, (18) holds and $\xi_{2}= \pm 1, \xi_{4}= \pm 1$. From (14) and (18) we get 


$$
\left\{\begin{array}{l}
x_{0}+x_{4}+x_{8}=1, \\
x_{1}+x_{5}+x_{9}=0, \\
x_{2}+x_{6}+x_{10}=0, \\
x_{3}+x_{7}+x_{11}=0 .
\end{array}\right.
$$

From $\xi_{2}= \pm 1$ we get

$$
\left\{\begin{array}{c}
2 x_{0}+x_{1}-x_{2}-2 x_{3}-x_{4}+x_{5}+2 x_{6}+x_{7}-x_{8} \\
-2 x_{9}-x_{10}+x_{11}=2 \rho_{1}, \\
x_{1}+x_{2}-x_{4}-x_{5}+x_{7}+x_{8}-x_{10}-x_{11}=0,
\end{array}\right.
$$

and from $\xi_{4}= \pm 1$ :

$$
\left\{\begin{array}{c}
2 x_{0}-x_{1}-x_{2}+2 x_{3}-x_{4}-x_{5}+2 x_{6}-x_{7}-x_{8} \\
\quad+2 x_{9}-x_{10}-x_{11}=2 \rho_{2}, \\
x_{1}-x_{2}+x_{4}-x_{5}+x_{7}-x_{8}+x_{10}-x_{11}=0 .
\end{array}\right.
$$

Solving (19), (20), (21) simultaneously and remembering that the variables are integers, we get $\rho_{1}=\rho_{2}=1, x_{1}=-x_{7}, x_{2}=x_{0}+x_{4}-1$, $x_{3}=x_{5}-x_{7}, x_{6}=1-x_{0}, x_{8}=1-x_{0}-x_{4}, x_{9}=x_{7}-x_{5}, x_{10}=-x_{4}, x_{11}=$ $-x_{5}$. Then for $M=X X^{T}$ we must have $7-4.3^{1 / 2}=\xi_{1} \bar{\xi}_{1}$. Using (15) this becomes

$$
\begin{gathered}
\left(3 x_{0}-2\right)^{2}+3\left(x_{5}+x_{7}\right)^{2}+9\left(x_{5}-x_{7}\right)^{2}+3\left(x_{0}+2 x_{4}-1\right)^{2}=7, \\
-2\left(x_{5}+x_{7}\right)\left(3 x_{0}-2\right)+6\left(x_{5}-x_{7}\right)\left(x_{0}+2 x_{4}-1\right)=-4 .
\end{gathered}
$$

From (22) we first obtain $x_{5}=x_{7}$, then $x_{5}=x_{7}=0$. But then we contradict (23). Hence the $G$-class number is two.

7. The alternating group of order twelve. This group is generated by elements $a, b, c$ with $a^{2}=b^{2}=c^{3}=1, a b=b a, a c=c a b$, $b c=c a$. The irreducible constituents of the group matrix $X$ are most easily computed if we take the group elements in the order, $1, a, b, a b, c, c a, c b, c a b, c^{2}, c^{2} a, c^{2} b, c^{2} a b$. Then the group matrix partitions into $4 \times 4$ blocks each of which has the structure of

$$
N=\left[\begin{array}{llll}
\alpha & \beta & \gamma & \delta \\
\beta & \alpha & \delta & \gamma \\
\gamma & \delta & \alpha & \beta \\
\delta & \gamma & \beta & \alpha
\end{array}\right]
$$

If $V$ denotes the unitary matrix of (3), then $V N V^{*}=\operatorname{diag}(\alpha+\beta+$ $\gamma+\delta, \alpha+\beta-\gamma-\delta, \alpha-\beta+\gamma-\delta, \alpha-\beta-\gamma+\delta)$. Thus each block in $X$ can be diagonalized. After the same permutation of rows and 
columns, the group matrix splits up into a direct sum of four $3 \times 3$ blocks, of which one is a circulant and may be diagonalized. Let $\left(x_{0}, x_{1}, \cdots, x_{11}\right)^{T}$ be the first column of $X$.

Let $\eta_{1}=x_{0}+x_{1}+x_{2}+x_{3}, \eta_{2}=x_{4}+x_{5}+x_{6}+x_{7}, \eta_{3}=x_{8}+x_{9}+$ $x_{10}+x_{11}, a_{11}=x_{2}+x_{3}, a_{22}=x_{1}+x_{3}, a_{33}=x_{1}+x_{2}, a_{12}=x_{9}+x_{11}, a_{23}=$ $x_{9}+x_{10}, a_{31}=x_{10}+x_{11}, a_{13}=x_{5}+x_{6}, a_{21}=x_{6}+x_{7}, a_{32}=x_{5}+x_{7}$. Also now let $\omega=\left(-1+(-3)^{1 / 2}\right) / 2$. Define $\varepsilon_{1}, \varepsilon_{2}, \varepsilon_{3}, A_{x}$ by:

$$
\begin{gathered}
{\left[\begin{array}{rrr}
3^{-1 / 2} & 3^{-1 / 2} & 3^{-1 / 2} \\
3^{-1 / 2} & \omega 3^{-1 / 2} & \omega^{2} 3^{-1 / 2} \\
3^{-1 / 2} & \omega^{2} 3^{-1 / 2} & \omega 3^{-1 / 2}
\end{array}\right]\left[\begin{array}{l}
\eta_{1} \\
\eta_{2} \\
\eta_{3}
\end{array}\right]=3^{-1 / 2}\left[\begin{array}{l}
\varepsilon_{1} \\
\varepsilon_{2} \\
\varepsilon_{3}
\end{array}\right],} \\
A_{X}=\left[\begin{array}{lll}
\eta_{1}-2 a_{11} & \eta_{3}-2 a_{12} & \eta_{2}-2 a_{13} \\
\eta_{2}-2 a_{21} & \eta_{1}-2 a_{22} & \eta_{3}-2 a_{23} \\
\eta_{3}-2 a_{31} & \eta_{2}-2 a_{32} & \eta_{1}-2 a_{33}
\end{array}\right] .
\end{gathered}
$$

Then there exists a unitary $U$ such that $U X U^{*}=\left(\varepsilon_{1}\right)+\left(\varepsilon_{2}\right)+\left(\varepsilon_{3}\right) \dot{+}$ $A_{X}+A_{X}+A_{X}$. Moreover $X$ is unimodular if and only if $\operatorname{det} A_{X}=$ \pm 1 and $\varepsilon_{1}, \varepsilon_{2}, \varepsilon_{3}$ are units in $R(\omega)$. Thus $\varepsilon_{1}, \varepsilon_{2}, \varepsilon_{3}$ have to be roots of unity and since the matrix in (24) is unitary, this forces $\eta_{1}^{2}+\eta_{2}^{2}+$ $\eta_{3}^{2}=\left(\left|\varepsilon_{1}\right|^{2}+\left|\varepsilon_{2}\right|^{2}+\left|\varepsilon_{3}\right|^{2}\right) / 3=1$. Thus exactly one of $\eta_{1}, \eta_{2}, \eta_{3}$ is \pm 1 , the other two are zero. Note that $a_{11}=x_{2}+x_{3}, a_{22}=x_{1}+x_{3}, a_{33}=$ $x_{1}+x_{2}$, possess an integral solution $x_{1}, x_{2}, x_{3}$ if and only if $a_{11}+a_{22}+$ $a_{33} \equiv 0(\bmod 2)$; a similar remark holds for $a_{12}, a_{23}, a_{31}$; and for $a_{13}, a_{21}, a_{32}$. Thus $X$ is $i u$ if and only if $A_{X}$ is $i u$ and exactly two of $\eta_{1}, \eta_{2}, \eta_{3}$ are zero and one is \pm 1 , and $a_{11}+a_{22}+a_{33} \equiv a_{12}+a_{23}+a_{31} \equiv a_{13}+a_{21}+$ $a_{32} \equiv 0(\bmod 2)$. The pdsiu $X$ arise when $\varepsilon_{1}=\varepsilon_{2}=\varepsilon_{3}=1, \eta_{1}=1, \eta_{2}=$ $\eta_{3}=0, A_{X}$ is pdsiu.

Now if $Y, Z$ are pdsiu group matrices we have $Z=X Y X^{T}$ if and only if $A_{z}=A_{X} A_{Y} A_{X}^{T}$ and $\varepsilon_{i}(z)=\varepsilon_{i}(X) \varepsilon_{i}(Y) \overline{\varepsilon_{i}(X)}, i=1,2,3$. This last condition is met since $\varepsilon_{i}(X) \overline{\varepsilon_{i}(X)}=1$ because $\varepsilon_{i}(X)$ is a root of unity. The fact that $A_{Y}$ is pdsiu and the fact that the $C$-class number is one at $n=3$ implies that $A_{T}=W W^{T}$ for some iu $W$. Here $W$ need not be an $A_{X}$. Consider $W \bmod 2$. Since $\bmod 2, A_{Y} \equiv I_{3}, W(\bmod 2)$ is orthogonal. Hence, mod $2, W$ is a permutation matrix. We may find a $3 \times 3$ permutation matrix $Q$ such that, $\bmod 2, W Q \equiv I_{3}$. We can do more. If we permit $Q$ to be a generalized permutation matrix (nonzero entries are \pm 1$)$ we can force $W Q \equiv I_{3}(\bmod 2)$ and each diagonal element of $W Q$ is $\equiv 1(\bmod 4)$. Changing notation and letting $W Q$ be $W$, we have $A_{Y}=W W^{T}$ where now $W$ is $i u$ and $(\bmod 4)$ has 1 in each diagonal position and $(\bmod 4)$ has 0 or 2 in each off-diagonal position. Now one can write down all 64 matrices $W(\bmod 4)$ of this type and determine those for which $W W^{T}$ has the structure $(\bmod 4)$ 
of an $A_{Y}$. It turns out that the $W$ matrices $(\bmod 4)$ with this property are precisely the $W$ matrices with an even number of twos $(\bmod 4)$ off the main diagonal. Certain of these acceptable $W$ already have the structure $(\bmod 4)$ of an $A_{Y}$. When this is so, $Y$ is in the principal $G$-class. For all those acceptable $W$ not $(\bmod 4)$ of the form of an $A_{x}$, it turns out that $W T$, where

$$
T=\left[\begin{array}{lll}
1 & 2 & 2 \\
0 & 1 & 0 \\
0 & 0 & 1
\end{array}\right]
$$

is an $A_{X}$. Let $H=T^{-1}\left(T^{-1}\right)^{T}$. Then $A_{Y}=(W T) H(W T)^{T}=A_{X} H A_{X}^{T}$ where $A_{X}=W T$. Moreover, $H$ is an $A_{Z}$. Thus $Y$ is in the same $G$-class as $Z$, where $A_{Z}=H$. Is $Z$ in the principal $G$-class? If so $H=A_{X} A_{X}^{T}$ for some $X$. But it is easy to find all integral $B$ for which $H=B B^{T}$; none is $(\bmod 4)$ an $A_{X}$. Hence the $G$-class number is two.

8. The dihedral group of order twelve. As is $\S 4$ the group matrix may be taken to have the form (2) with $C=B^{T}, D=A^{T}$. Let $A=x_{0} I_{6}+x_{1} P_{6}+\cdots+x_{5} P_{6}^{5}, B=x_{6} I_{6}+x_{7} P_{6}+\cdots+x_{11} P_{6}^{5}$. There exists a unitary $U$ such that $U X U^{*}=\left(\varepsilon_{1}\right)+\left(\varepsilon_{2}\right)+\left(\varepsilon_{3}\right)+\left(\varepsilon_{4}\right)+X_{1} \dot{+}$ $X_{1}+X_{2}+X_{2}$ where: if $\eta_{1}=x_{0}+x_{2}+x_{4}, \eta_{2}=x_{1}+x_{3}+x_{5}, \eta_{3}=x_{6}+$ $x_{8}+x_{10}, \eta_{4}=x_{7}+x_{9}+x_{11}$, and if $a=x_{0}+x_{3}, b=x_{1}+x_{4}, \alpha=x_{0}-x_{3}$, $\beta=x_{4}-x_{1}, c=x_{6}+x_{9}, d=x_{7}+x_{10}, \gamma=x_{6}-x_{9}, \delta=x_{10}-x_{7}$, then (3) holds, and, in addition,

$$
X_{1}=\left[\begin{array}{cc}
X_{1,1} & \bar{X}_{1,2} \\
X_{1,1} & \bar{X}_{1,2}
\end{array}\right], \quad X_{2}=\left[\begin{array}{cc}
X_{2,1} & \bar{X}_{2,2} \\
X_{2,2} & \bar{X}_{2,1}
\end{array}\right]
$$

where

$$
\left\{\begin{array}{l}
X_{1,1}=\left(3 a-\eta_{1}-\eta_{2}+(-3)^{1 / 2}\left(a+2 b-\eta_{1}-\eta_{2}\right)\right) / 2, \\
X_{1,2}=\left(3 c-\eta_{3}-\eta_{4}+(-3)^{1 / 2}\left(c+2 d-\eta_{3}-\eta_{4}\right)\right) / 2, \\
X_{2,1}=\left(3 \alpha-\eta_{1}+\eta_{2}+(-3)^{1 / 2}\left(\eta_{1}-\eta_{2}-\alpha-2 \beta\right)\right) / 2, \\
X_{2,2}=\left(3 \gamma-\eta_{3}+\eta_{4}+(-3)^{1 / 2}\left(\eta_{3}-\eta_{4}-\gamma-2 \delta\right)\right) / 2 .
\end{array}\right.
$$

Note that $x_{0}, \cdots, x_{11}$ are integers if and only if $a \equiv \alpha, b \equiv \beta, c \equiv \gamma$, $d \equiv \delta(\bmod 2)$. As $\varepsilon_{1}, \varepsilon_{2}, \varepsilon_{3}, \varepsilon_{4}$, det $X_{1}$, det $X_{2}$ are rational integers, $X$ is unimodular if and only if $\varepsilon_{1}, \varepsilon_{2}, \varepsilon_{3}, \varepsilon_{4}$, det $X_{1}$, det $X_{2}$ are each \pm 1 . Hence, as with the dihedral group of order eight, exactly one of $\eta_{1}, \eta_{2}, \eta_{3}, \eta_{4}$ is \pm 1 and the other three are zero. By considering the formulas for $\operatorname{det} X_{1}$ and $\operatorname{det} X_{2}(\bmod 3)$, we find $\operatorname{det} X_{1}=\operatorname{det} X_{2}=1$ if $\eta_{1}$ or $\eta_{2}$ is \pm 1 , and $\operatorname{det} X_{1}=\operatorname{det} X_{2}=-1$ if $\eta_{3}$ or $\eta_{4}$ is \pm 1 . The $p d$ siu group matrices arise when $\eta_{1}=1$ and $X_{1,1}$ and $X_{2,1}$ are real and positive. If $\eta_{1}$ or $\eta_{2}$ is \pm 1 we let $X_{1,1}=\left(A_{X}+(-3)^{1 / 2} B_{X}\right) / 2, X_{1,2}=\left(C_{X}+(-3)^{1 / 2} D_{X}\right) / 2$, 
$X_{2,1}=\left(\mathfrak{Y}_{X}+(-3)^{1 / 2} \mathfrak{B}_{X}\right) / 2, X_{2,2}=\left(\mathfrak{E}_{X}+(-3)^{1 / 2} \mathfrak{D}_{X}\right) / 2$; and if $\eta_{3}$ or $\eta_{4}$ is \pm 1 we let $X_{1,1}=\left(C_{X}+(-3)^{1 / 2} D_{X}\right) / 2, X_{1,2}=\left(A_{X}+(-3)^{1 / 2} B_{X}\right) / 2, X_{2,1}=$ $\left(\mathfrak{S}_{X}+(-3)^{1 / 2} \mathfrak{D}_{X}\right) / 2, X_{2,2}=\left(\mathfrak{A}_{X}+(-3)^{1 / 2} \mathfrak{B}_{X}\right) / 2$.

Now let $Z, Y$ are pdsiu group matrices; then $Z=X Y X^{T}$ holds if and only if $\varepsilon_{i}(Z)=\varepsilon_{i}(X) \varepsilon_{i}(Y) \overline{\varepsilon_{i}(X)}$ for $i=1,2,3,4, Z_{1}=X_{1} Y_{1} X_{1}^{*}$, $Z_{2}=X_{2} Y_{2} X_{2}^{*}$. The first of these conditions need not concern us as $\varepsilon_{i}(X)$ is always to be \pm 1 . We proceed to show that, given $Y$, we can choose $X i u$ such that $Z_{2}=I_{2}$. If $Y_{2}=I_{2}$ we have nothing to do. Otherwise we compute as in Lemma 2 that

$$
\begin{aligned}
2\left(A_{Z}-A_{Y}\right)= & A_{Y}\left(C_{X}^{2}+3 D_{X}^{2}\right) \\
& +C_{Y}\left(A_{X} C_{X}-3 B_{X} D_{X}\right)+3 D_{Y}\left(A_{X} D_{X}+B_{X} C_{X}\right), \\
2\left(\mathfrak{A}_{Z}-\mathfrak{A}_{Y}\right)= & \mathfrak{A}_{Y}\left(\mathfrak{C}_{X}^{2}+3 \mathfrak{D}_{X}^{2}\right) \\
& +\mathfrak{C}_{Y}\left(\mathfrak{U}_{X} \mathfrak{\complement}_{X}-3 \mathfrak{B}_{X} \mathfrak{D}_{X}\right)+3 \mathfrak{D}_{Y}\left(\mathfrak{A}_{X} \mathfrak{D}_{X}+\mathfrak{B}_{X} \mathfrak{\complement}_{X}\right) .
\end{aligned}
$$

We now assign special values to the quantities entering into $X$. If we put $\eta_{1}=-\rho_{1}, \eta_{2}=\eta_{3}=\eta_{4}=0, a=\alpha=\rho_{1}, b=\beta=-\rho_{1}, c=\gamma=\rho_{2}$, $d=\delta=-\rho_{2}$ then we get $A_{X}=\mathfrak{A}_{X}=4 \rho_{1}, B_{X}=\mathfrak{B}_{X}=0, C_{X}=\mathfrak{S}_{X}=3 \rho_{2}$ $D_{X}=-\rho_{2}, \mathfrak{D}_{X}=\rho_{2}$. For this $i u X, \mathfrak{A}_{Z}-\mathfrak{A}_{Y}<0$ will hold if

$$
\mathfrak{A}_{Y}+\rho_{1} \rho_{2} \mathfrak{E}_{Y}+\rho_{1} \rho_{2} \mathfrak{D}_{Y}<0 .
$$

Next we put $\eta_{1}=\rho_{1}, \eta_{2}=\eta_{3}=\eta_{4}=0, a=\alpha=\rho_{1}, b=\beta=\rho_{2}, c=\gamma=\rho_{3}$, $d=\delta=-\rho_{3}$. Then $A_{X}=\mathfrak{A}_{X}=2 \rho_{1}, B_{X}=2 \rho_{2}, \mathfrak{B}_{X}=-2 \rho_{2}, C_{X}=\mathfrak{c}_{X}=3 \rho_{3}$, $D_{x}=-\rho_{2}, D_{X}=-\rho_{3}, \mathfrak{D}_{X}=\rho_{3}$. For this $i u X, \mathfrak{A}_{Z}-\mathfrak{A}_{Y}<0$ will hold if

$$
12 \mathfrak{A}_{Y}+\mathfrak{C}_{Y}\left(6 \rho_{1} \rho_{3}+6 \rho_{2} \rho_{3}\right)+3 \mathfrak{D}_{Y}\left(2 \rho_{1} \rho_{3}-6 \rho_{2} \rho_{3}\right)<0 .
$$

If $\rho_{1}=\rho_{2}$ this becomes

$$
\mathfrak{A}_{Y}+\rho_{1} \rho_{3} \mathfrak{E}_{Y}-\rho_{1} \rho_{3} \mathfrak{D}_{Y}<0,
$$

and if $\rho_{1}=-\rho_{2}$ this becomes

$$
\mathfrak{U}_{Y}+2 \rho_{1} \rho_{3} \mathfrak{D}_{Y}<0 .
$$

Choosing the signs $\rho_{1}, \rho_{2}, \rho_{3}$ suitably, (29) and (30) becomes

$$
\mathfrak{A}_{Y}-\left|\mathfrak{E}_{Y}\right|-\left|\mathfrak{D}_{Y}\right|<0,
$$

and (31) becomes

$$
\mathfrak{A}_{Y}-2\left|\mathfrak{D}_{Y}\right|<0 \text {. }
$$

So we can make $\mathfrak{A}_{Z}<\mathfrak{A}_{Y}$ if $\mathfrak{A}_{Y}, \mathfrak{\complement}_{Y}, \mathfrak{D}_{Y}$ satisfy either (32) or (33). As in $\S 4$, the facts that $\mathfrak{A}_{Y}>0$ and $\mathfrak{A}_{Y}^{2}=4+\mathfrak{C}_{Y}^{2}+3 \mathfrak{D}_{Y}^{2}$ show that (32) and (33) are equivalent to

$$
2+\left|\mathfrak{D}_{Y}\right|^{2}-\left|\mathfrak{C}_{Y}\right|\left|\mathfrak{D}_{Y}\right|<0,
$$




$$
4+\left|\mathfrak{S}_{Y}\right|^{2}-\left|\mathfrak{D}_{Y}\right|^{2}<0,
$$

respectively.

Now the region in the positive quadrant of the $\mathbb{C}_{Y}, \mathfrak{D}_{Y}$ plane satisfying neither (34) nor (35) is a region of infinite extent with hyperbolas as part of the boundary. Remembering that $\mathfrak{s}_{Y} \equiv 0(\bmod 3)$, we find several points $\left(\left|\mathfrak{C}_{Y}\right|,\left|\mathfrak{D}_{Y}\right|\right)$ in our region: $\left(\left|\mathfrak{C}_{Y}\right|,\left|\mathfrak{D}_{Y}\right|\right)=(0,2)$, $(3,1),(3,2)$ and points with $\left|\mathfrak{C}_{Y}\right|=\left|\mathfrak{D}_{Y}\right|$ and points with $\mathfrak{D}_{Y}=0$. The points $(0,2),(3,1),(3,2)$ give $\mathfrak{A}_{T}=4$ or 5 and this can be rejected on the grounds that a pdsiu $Y$ has $\mathfrak{B}_{Y}=0, \eta_{1}=1$ and then $A_{Y}=4$ or 5 give a nonintegral $\alpha, \beta$. The cases in which $\mathfrak{D}_{Y}=0$ or $\left|\mathfrak{C}_{Y}\right|=\left|\mathfrak{D}_{Y}\right|$ are rejected by showing that $\mathfrak{U}_{Y}^{2}=4+\mathfrak{C}_{Y}^{2}+3 \mathfrak{D}_{Y}^{2}$ does not give a positive integral $\mathfrak{A}_{Y}$, except if $\mathfrak{S}_{Y}=\mathfrak{D}_{Y}=0, \mathfrak{A}_{Y}=2$. When $\mathfrak{夭}_{Y}=\mathfrak{D}_{Y}=0$, $A_{Y}=2$, we have $Y_{2}=I_{2}$. Thus we have shown that if $Y_{2} \neq I_{2}$ then we can find an $i u X$ so that $\mathfrak{A}_{Z}<\mathfrak{I}_{Y}$. Since $\mathfrak{A}_{Z}>0$, eventually this descent halts and then $Z_{2}=I_{2}$.

Thus assume $Y_{2}=I_{2}$. Our next goal is, using only $X$ for which $X_{2} X_{2}^{*}=I_{2}$, to make $A_{Z}<A_{Y}$. Notice that $Y_{2}=I_{2}$ and $\eta_{1}=1$ implies that the parameters $\alpha, \beta, \gamma, \delta$ of $Y_{2}$ are $\alpha=1, \beta=\gamma=\delta=0$. Thus the parameters $a, b, c, d$ of $Y$ satisfy $a \equiv 1, b \equiv c \equiv d \equiv 0(\bmod 2)$. Hence $C_{Y} \equiv 0(\bmod 6)$ and $D_{Y} \equiv c \equiv-c \equiv C_{Y}(\bmod 4)$. We next determine those $X$ for which $X_{2} X_{2}^{*}=I_{2}$. By Lemma 2 these $X$ must have $\mathfrak{夭}_{X}=\mathfrak{D}_{X}=0$, so that $\mathfrak{A}_{X}^{2}+3 \mathfrak{B}_{X}^{2}=4, \mathfrak{A}_{X}= \pm 2, \mathfrak{B}_{X}=0$, or $\mathfrak{A}_{X}=$ $\pm 1, \mathfrak{B}_{x}= \pm 1$. It is then easy to determine the parameters $\alpha, \beta, \gamma, \delta$ of $X$. We find that if $\eta_{1}$ or $\eta_{2}$ is \pm 1 then $\gamma=\delta=0$ and not both $\alpha, \beta$ are odd; and if $\eta_{3}$ or $\eta_{4}$ is \pm 1 then $\alpha=\beta=0$ and not both $\gamma, \delta$ are odd. So in $X$ the parameters $a, b, c, d$ are restricted by: both $c, d$ are even and not both $a, b$ are odd in the cases when $\eta_{1}$ or $\eta_{2}$ is \pm 1 ; and both $a, b$ are even and not both $c, d$ are odd in the cases when $\eta_{3}$ or $\eta_{4}$ is \pm 1 . In particular if we put $\eta_{1}=-\rho_{1}, \alpha=0, \beta=-\left(\rho_{1}+\rho_{2}\right) / 2$, $\gamma=0, \delta=0$, or if we put $\eta_{1}=\rho_{1}, \alpha=\rho_{1}, \beta=\gamma=\delta=0$, then $X_{2} X_{2}^{*}=I_{2}$.

We now seek $X$ for which $A_{Z}<A_{Y}$ and $X_{2} X_{2}^{*}=I_{2}$. To this end we give special values to the parameters in $X$. Put $\eta_{1}=\rho_{1}, \eta_{2}=\eta_{3}=$ $\eta_{4}=0, a=\rho_{1}, \alpha=\rho_{1}, b=-2 \rho_{2}, \beta=0, \gamma=c=0, d=2 \rho_{4}, \delta=0$. Then $A_{X}=2 \rho_{1}, B_{X}=-4 \rho_{2}, C_{X}=0, D_{X}=4 \rho_{4}, X$ is $i u$ and $X_{2} X_{2}^{*}=I_{2}$. From (27) we find that the signs $\rho_{1}, \rho_{2}, \rho_{4}$ can be chosen to make $A_{Z}<A_{Y}$ if

$$
2 A_{Y}-2\left|C_{Y}\right|-\left|D_{Y}\right|<0 \text {. }
$$

Next set $\eta_{1}=-\rho_{1}, \alpha=-2 \rho_{1}, \alpha=0, b=\left(\rho_{1}-3 \rho_{2}\right) / 2, \beta=-\left(\rho_{1}+\rho_{2}\right) / 2$, $\gamma=c=0, d=2 \rho_{1}, \delta=0$. Then $A_{X}=-5 \rho_{1}, B_{X}=-3 \rho_{2}, C_{X}=0, D_{X}=$ $4 \rho_{4}, X$ is $i u$ and $X_{2} X_{2}^{*}=I_{2}$. Then from (27) we can choose the signs $\rho_{1}, \rho_{2}, \rho_{4}$ so that $A_{Z}<A_{Y}$ if 


$$
4 A_{Y}-3\left|C_{Y}\right|-5\left|D_{Y}\right|<0 \text {. }
$$

Finally we set $\eta_{1}=-\rho_{1}, a=2 \rho_{1}, \alpha=0, b=\left(\rho_{2}-3 \rho_{1}\right) / 2, \beta=-\left(\rho_{1}+\rho_{2}\right) / 2$, $c=\gamma=0, d=2 \rho_{4}, \delta=0$. Then $A_{X}=7 \rho_{1}, B_{X}=\rho_{2}, C_{X}=0, D_{X}=4 \rho_{4}$. We can, using (27), choose the signs $\rho_{1}, \rho_{2}, \rho_{4}$ so that $A_{Z}<A_{Y}$ if

$$
4 A_{Y}-\left|C_{Y}\right|-7\left|D_{Y}\right|<0 \text {. }
$$

Using $A_{Y}>0, A_{Y}^{2}=4+C_{Y}^{2}+3 D_{Y}^{2}$, we find that (36), (37), (38) are equivalent to

$$
\begin{gathered}
16+11 D_{Y}^{2}-4\left|C_{Y}\right|\left|D_{Y}\right|<0, \\
64+7 C_{Y}^{2}+23 D_{Y}^{2}-30\left|C_{Y}\right|\left|D_{Y}\right|<0, \\
64+15 C_{Y}^{2}-D_{Y}^{2}-14\left|C_{Y}\right|\left|D_{Y}\right|<0,
\end{gathered}
$$

respectively.

Now the region in the positive quadrant of the $C_{Y}, D_{Y}$ plane not satisfying any of (39), (40), (41) is a region of infinite extent with a portion of three hyperbolas as part of the boundary. In this region the only points $\left(\left|C_{Y}\right|,\left|D_{Y}\right|\right)$ with $C_{Y} \equiv 0(\bmod 6), C_{Y} \equiv D_{Y}(\bmod 4)$ are $(0,4),(6,2),(0,8),(12,4)$, together with points for which $\left|C_{Y}\right|=\left|D_{Y}\right|$ or for which $D_{Y}=0$. We can reject $(0,4)$ and $(6,2)$ since, using $A_{Y}^{2}=$ $4+C_{Y}^{2}+3 D_{Y}^{2}$, they give nonintegral $A_{Y}$. Now $\left|C_{Y}\right|=\left|D_{Y}\right|$ gives $A_{Y}^{2}=4+4 D_{Y}^{2}$, so $\left(A_{Y}-2 D_{Y}\right)\left(A_{Y}+2 D_{Y}\right)=4$. This gives a finite number of possibilities of which only $C_{Y}=D_{Y}=0, A_{Y}=2$ is acceptable. Similarly $D_{Y}=0$ leads only to $C_{Y}=D_{Y}=0, A_{Y}=2$. Now $A_{Y}=2$, $C_{Y}=D_{Y}=0$ gives $Y_{1}=I_{2}$. Thus, subject to the constraint that $Z_{2}=Y_{2}=I_{2}$ we have found iu $X$ so that in $Z=X Y X^{T}$ we have $A_{Z}<A_{Y}$. Since this descent must eventually stop, we have shown that any pdsiu group matrix is in the $G$ class of $I_{12}$ or the $G$-class of a group matrix $Y$ for which $Y_{2}=I_{2}, A_{Y}=14,\left(C_{Y}, D_{Y}\right)=(0, \pm 8)$ or $( \pm 12, \pm 4)$. Let now $Y$ be the pdsiu group matrix for which $Y_{2}=I_{2}$, $A_{Y}=14, C_{Y}=0, D_{Y}=8$. We now exhibit iu $X$ for which $Z=X Y X^{T}$ has $Z_{2}=I_{2}, A_{Z}=14,\left(C_{Z}, D_{Z}\right)=(0,-8)$ or $( \pm 12, \pm 4)$.

First put $\eta_{1}=-\rho_{1}, \alpha=0, \alpha=0, b=-\left(\rho_{1}+\rho_{2}\right) / 2, \beta=-\left(\rho_{1}+\rho_{2}\right) / 2$, $c=\gamma=0, d=\delta=0$. Then $A_{X}=\rho_{1}, B_{X}=-\rho_{2}, C_{X}=D_{X}=0, X_{2} X_{2}^{*}=I_{2}$, and $A_{Z}=14, C_{Z}=-12 \rho_{1} \rho_{2}, D_{Z}=-4$. Next put $\eta_{1}=-\rho_{1}, a=2 \rho_{1}$, $\alpha=0, b=\left(\rho_{2}-3 \rho_{1}\right) / 2, \beta=-\left(\rho_{1}+\rho_{2}\right) / 2, c=0, \gamma=0, d=-2 \rho_{1}, \delta=0$. Then $A_{X}=7 \rho_{1}, B_{X}=\rho_{2}, C_{X}=0, D_{X}=-4 \rho_{1}, X_{2} X_{2}^{*}=I_{2}, A_{Z}=14, C_{Z}=0$, $D_{z}=-8$. Finally put $\eta_{3}=-\rho_{1}, \alpha=\alpha=b=\beta=c=\gamma=0, d=\delta=$ $-\left(\rho_{1}+\rho_{2}\right) / 2$. Then $A_{X}=\rho_{1}, B_{X}=-\rho_{2}, C_{X}=D_{X}=0, \mathfrak{A}_{X}=\rho_{1}, \mathfrak{B}_{X}=\rho_{2}$, $\mathfrak{c}_{X}=\mathfrak{D}_{X}=0$. Moreover $X_{2} X_{2}^{*}=I_{2}$ and $Z_{1}=X_{1} Y_{1} X_{1}^{*}$ has $A_{Z}=14$, $C_{z}=-12 \rho_{1} \rho_{2}, D_{z}=4$. 
We have thus established that the $G$-class number is at most two. If it were one there would be an $X$ for which $X_{1} Y_{1} X_{1}^{*}=I_{2}$ and $X_{2} X_{2}^{*}=I_{2}$. The second condition forces (as previously noted): $\gamma=\delta=0$ or $\alpha=\beta=0$. In turn these as before, $C_{X} \equiv 0(\bmod 6), C_{X} \equiv D_{X}(\bmod 4)$. Then Lemma 2 shows that $C_{X}^{2}+3 D_{X}^{2}<C_{Y}^{2}+3 D_{Y}^{2}=192$. Using $A_{X}^{2}+3 B_{X}^{2}=4+C_{X}^{2}+3 D_{X}^{2}$, all possible values of $A_{X}, B_{X}, C_{X}, D_{X}$ are easily found and tested in (27). In all cases $A_{Z}-A_{Y} \geqq 0$. Thus we have proved that the $G$-class number is precisely two.

9. The group $a^{4}=1, b^{3}=1, a^{-1} b a=b^{2}$, of order twelve. If we take the group elements in the order $1, b, b^{2}, a, a b, a b^{2}, a^{2}, a^{2} b, a^{2} b^{2}, a^{3}, a^{3} b, a^{3} b^{2}$, then the group matrix $X$ partitions into blocks which are $3 \times 3$ circulants. Let $\left(x_{0}, x_{1}, \cdots, x_{11}\right)^{t^{\prime}}$ be the first column of $X$. We compute the irreducible representations as indicated in $\$ 2$. At one point it is necessary to make use of the following fact:

$$
2^{-1 / 2}\left[\begin{array}{rr}
I_{2} & I_{2} \\
I_{2} & -I_{2}
\end{array}\right]\left[\begin{array}{ll}
A & B \\
B & A
\end{array}\right] 2^{-1 / 2}\left[\begin{array}{rr}
I_{2} & I_{2} \\
I_{2} & -I_{2}
\end{array}\right]=\left[\begin{array}{cc}
A+B & 0 \\
0 & A-B
\end{array}\right]
$$

if $A, B$ are $2 \times 2$ matrices. Thus we find a unitary $U$ such that $U X U^{*}=\left(\varepsilon_{1}\right)+\left(\varepsilon_{4}\right)+\left(\varepsilon_{2}\right)+\left(\varepsilon_{3}\right)+X_{1}+X_{1}+X_{2}+X_{2}$. Here, if $\eta_{1}=$ $x_{0}+x_{1}+x_{2}, \eta_{2}=x_{6}+x_{7},+x_{8}, \eta_{3}=x_{3}+x_{4}+x_{5}, \eta_{4}=x_{9}+x_{10}+x_{11}$, then:

$$
\left[\begin{array}{rrrr}
1 / 2 & 1 / 2 & 1 / 2 & 1 / 2 \\
1 / 2 & i / 2 & -1 / 2 & -i / 2 \\
1 / 2 & -1 / 2 & 1 / 2 & -1 / 2 \\
1 / 2 & -i / 2 & -1 / 2 & i / 2
\end{array}\right]\left[\begin{array}{l}
\eta_{0} \\
\eta_{4} \\
\eta_{2} \\
\eta_{3}
\end{array}\right]=\frac{1}{2}\left[\begin{array}{l}
\varepsilon_{1} \\
\varepsilon_{4} \\
\varepsilon_{2} \\
\varepsilon_{3}
\end{array}\right] .
$$

The matrix $X_{1}$ is described by (25) and (26) where $a=x_{0}+x_{6}, b=x_{2}+x_{8}$, $c=x_{3}+x_{9}, d=x_{5}+x_{11} \cdot \quad X_{2}$ is described by

$$
X_{2}=\left[\begin{array}{rr}
X_{2,1} & -\bar{X}_{2,2} \\
X_{2,2} & \bar{X}_{2,1}
\end{array}\right]
$$

with $X_{2,1}, X_{2,2}$ given by (26); $\alpha=x_{0}-x_{6}, \beta=x_{2}-x_{8}, \gamma=x_{3}-x_{9}$, $\delta=x_{5}-x_{11}$.

As before, for integral $x_{0}, x_{1}, \cdots, x_{11}$ we must have $a \equiv \alpha, b \equiv \beta$, $c \equiv \gamma, d \equiv \delta(\bmod 2)$. Here $\varepsilon_{1}, \varepsilon_{2}, \varepsilon_{3}, \varepsilon_{4}$, det $X_{1}$, det $X_{2}$ are algebraic integers and must be units if $X$ is to be $i u$. Since the $\varepsilon_{i}$ are Gaussian integers, this forces the $\varepsilon_{i}$ to be roots of unity. Because the matrix in (42) is unitary, this forces exactly one $\eta_{i}$ to be \pm 1 , the others to be zero. Now in fact $\operatorname{det} X_{1}$, $\operatorname{det} X_{2}$ are rational integers and $\operatorname{det} X_{2}>0$. Thus $\operatorname{det} X_{1}= \pm 1\left(+1\right.$ if $\eta_{1}$ or $\eta_{2}$ is $\pm 1,-1$ if $\eta_{3}$ or $\eta_{4}$ is \pm 1 ) and $\operatorname{det} X_{2}=1$. The pdsiu $X$ arise when $\eta_{1}=1, \varepsilon_{1}=\varepsilon_{2}=\varepsilon_{3}=\varepsilon_{4}=1$, $\operatorname{det} X_{1}=1, X_{1,1}>0, X_{2,1}>0$. From $\operatorname{det} X_{2}=1$ we get $\left|X_{2,1}\right|^{2}+\left|X_{2,2}\right|^{2}=1$. 
Each of $\left|X_{2,1}\right|^{2},\left|X_{2,2}\right|^{2}$ is a rational integer so either $X_{2,1}=0$ or $X_{2,2}=0$. When $X$ is pdsiu, $X_{2,1}$ is thus a positive unit in the field of $R\left((-3)^{1 / 2}\right)$, hence $X_{2,1}=1$ and hence $X_{2}=I_{2}$. But always if $X$ is just $i u$ we have $X_{2} X_{2}^{*}=I_{2}$. We show $X_{2,2}=0$ when $\eta_{1}$ or $\eta_{2}$ is \pm 1 ; and $X_{2,1}=0$ when $\eta_{3}$ or $\eta_{4}$ is \pm 1 . If we had $\eta_{1}$ or $\eta_{2}$ equal to \pm 1 and $X_{2,1}=0$ we would have $3 \alpha-\eta_{1}+\eta_{2}=0$, which is not true for any integer $\alpha$. Similarly if $\eta_{3}$ or $\eta_{4}$ is \pm 1 then $X_{2,2}=0$ is absurd. From this point on the discussion is almost word for word the same as the discussion in $\S 8$. We introduce $A_{X}, B_{X}, C_{X}, D_{X}, \mathfrak{A}_{X}, \mathfrak{B}_{X}, \mathfrak{夭}_{X}, \mathfrak{D}_{X}$ as in $\S 8$. We have just established that $\mathfrak{夭}_{X}=\mathfrak{D}_{X}=0$ and that $Y_{2}=I_{2}$ if $Y$ is pdsiu. We now carry on from the point in $\S 8$ at which we assumed $Y_{2}=I_{2}$. The conclusion we reach is that the $G$-class number is two.

10. The noncyclic abelian group of order twelve. By Theorem 2 the only pdsiu group matrix for this group is $I_{12}$.

11. Summary. Let $\Phi_{n}$ be the matrix on p. 331 of [5].

THEOREM 5. For all groups $G$ of order $n \leqq 13$, the G-class number is one, except for the cyclic groups of orders 8 and 12, the dihedral groups of orders 8 and 12 , the alternating group $A_{4}$, and the remaining nonabelian group of order twelve. In each of these exceptional cases the G-class number is two and the nonprincipal $G$-class is contained in the C-class of $\Phi_{n}$.

Acknowledgement. I have benefited from discussions of this problem with Dr. O. Taussky. In particular, through Dr. Taussky, I was aware of prior unpublished work of M. Kneser and E. C. Dade who computed the $G$-class number for the cyclic group of order nine (Kneser) and for a number of cyclic groups of prime order (Dade).

\section{REFERENCES}

1. G. Higman, The units of group rings, Proc. London Math. Soc. $\mathbf{1 6}$ (1940), 231-248.

2. M. Kneser, Klassenzahlen definiter Quadratischer Formen, Archiv der Math. 8 (1957), 241-250.

3. M. Newman, Circulant quadratic forms, Report of the Institute in the theory of numbers, Boulder, Colorado (1959), 189-193.

4. M. Newman and O. Taussky, Classes of definite unimodular circulants, Canad. Math. J. 9 (1956), 71-73.

5. O. T. O'Meara, Introduction to quadratic forms, Academic Press, New York, 1963.

6. O. Taussky, Matrices of rational integers, Bull. Amer. Math. Soc. 66 (1960), 327-345.

7. R. C. Thompson, Normal matrices and the normal basis in abelian number fields, Pacific J. Math. 12 (1962), 1115-1124.

8. R. C. Thompson Unimodular group matrices with rational integers as elements, Pacific J. Math. 14 (1964), 719-726. 


\section{PACIFIC JOURNAL OF MATHEMATICS}

\section{EDITORS}

\section{H. SAMELSON}

Stanford University

Stanford, California

R. M. Blumenthal

University of Washington

Seattle, Washington 98105

\author{
*J. DugundJI \\ University of Southern California \\ Los Angeles, California 90007 \\ RICHARD ARENS \\ University of California \\ Los Angeles, California 90024
}

\section{ASSOCIATE EDITORS}
E. F. BECKENBACH
B. H. NeUManN
F. WolF
K. YosIDA

\section{SUPPORTING INSTITUTIONS}

UNIVERSITY OF BRITISH COLUMBIA
CALIFORNIA INSTITUTE OF TECHNOLOGY
UNIVERSITY OF CALIFORNIA
MONTANA STATE UNIVERSITY
UNIVERSITY OF NEVADA
NEW MEXICO STATE UNIVERSITY
OREGON STATE UNIVERSITY
UNIVERSITY OF OREGON
OSAKA UNIVERSITY
UNIVERSITY OF SOUTHERN CALIFORNIA

UNIVERSITY OF BRITISH COLUMBIA

UNIVERSITY OF CALIFORNIA

MONTANA STATE UNIVERSITY

NEW MEXICO STATE UNIVERSITY

OREGON STATE UNIVERSITY

OSAKA UNIVERSITY

UNIVERSITY OF SOUTHERN CALIFORNIA

\author{
STANFORD UNIVERSITY \\ UNIVERSITY OF TOKYO \\ UNIVERSITY OF UTAH \\ WASHINGTON STATE UNIVERSITY \\ UNIVERSITY OF WASHINGTON \\ AMERICAN MATHEMATICAL SOCIETY \\ CHEVRON RESEARCH CORPORATION \\ TRW SYSTEMS \\ NAVAL ORDNANCE TEST STATION
}




\section{Pacific Journal of Mathematics \\ Vol. 17, No. $1 \quad$ January, 1966}

Carlos Jorge Do Rego Borges, On stratifiable spaces ................ 1

Felix Earl Browder, Topological methods for non-linear elliptic equations of

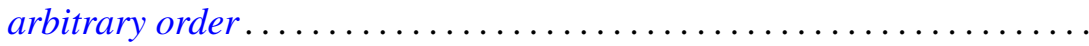

Gustave Choquet, Harry Corson and Victor Klee, Exposed points of convex

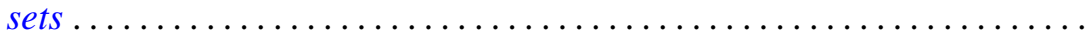

Phillip Emig, Remarks on the defect sum for a function meromorphic on an open Riemann surface ................................ 45

Ruth Goodman, A certain class of polynomials .................. 57

Sidney (Denny) L. Gulick, The bidual of a locally multiplicatively-convex

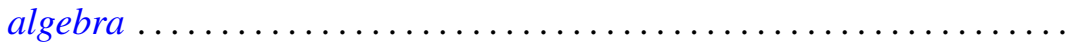

Eugene Carlyle Johnsen, Integral solutions to the incidence equation for finite projective plane cases of orders $n \equiv=2(\bmod 4) \ldots \ldots \ldots \ldots . .67$

Charles N. Kellogg, Centralizers and $H^{*}$-algebras .................. 121

Michael Lodato, On topologically induced generalized proximity relations. II .......................................... 131

P. H. Maserick, Half rings in linear spaces ..................... 137

Kathleen B O'Keefe, On a problem of J. F. Ritt .................... 149

Galen Lathrop Seever, Nonnegative projections on $C_{0}(X) \ldots \ldots \ldots \ldots$

Lawrence A. Shepp, Gaussian measures in function space ............ 167

Robert Charles Thompson, Classes of definite group matrices ........... 175 